\title{
Re-Classification of the Alleged Quasar Q0045-3337
}

\author{
Roberto Decarli ${ }^{1, *}$, Renato Falomo ${ }^{2}$, Jari Kotilainen ${ }^{3}$, Marzia Labita ${ }^{1}$, Riccardo Scarpa ${ }^{4}$ and Aldo \\ Treves $^{1}$
}

\author{
${ }^{1}$ Universita` degli Studi dell'Insubria, via Valleggio 11 I-22100 Como, Italy \\ ${ }^{2}$ INAF - Osservatorio Astronomico di Padova, Vicolo dell'Osservatorio 5 I-35122 Padova, Italy \\ ${ }^{3}$ Tuorla Observatory, University of Turku, Väisäläntie 20, FIN-21500 Piikkiö, Finland \\ ${ }^{4}$ Instituto de astrofisica de Canarias, via Lactea s/n 35, E-38205 La Laguna (Tenerife), Spain
}

\begin{abstract}
We present a medium-resolution optical spectrum of the alleged high-redshift quasar Q0045-3337, taken at the $\mathrm{ESO} / 3.6 \mathrm{~m}$ telescope. Our observations show that the object is not a quasar but a star of spectral type B. We suggest that the object is either a white dwarf or a halo population Blue Horizontal Branch star.
\end{abstract}

\section{INTRODUCTION}

We report on optical spectroscopic observations of the source Q0045-3337 (RA $\mathrm{R}_{\mathrm{J} 2000}$ : 0047 41.8, $\mathrm{DEC}_{\mathrm{J} 2000}$ : -33 20 55), that was classified [1] as a quasar (object 004517.0333717). In that work, the Automated Quasar Detection technique [2] was applied to objective prism plates. Due to the poor spectral resolution, the redshift $(\mathrm{z}=2.14)$ was determined only tentatively. The object is not flagged as a "good quality candidate", and no X-ray or radio counterparts were found. The target appears also in the Veron-Cetty \& Veron quasar catalog [3] (J004741.9-332055; V=18.75).

The source was imaged [4] in the Ks band with VLT/NACO using the adaptive optics technique, in a campaign aimed to observe host galaxies of high-z quasars that lie in the vicinity of bright stars $[4,5]$. No host galaxy was detected around the target, which appeared with $\mathrm{Ks}=18.75$ mag. A foreground spiral galaxy of unknown redshift and $\mathrm{Ks}=17.5 \mathrm{mag}$ was observed at $\sim 1.2 \mathrm{arcsec}$ from the target. Since the separation between Q0045-3337 and the galaxy is comparable to the estimated Einstein radius of the galaxy, it was argued that Q0045-3337 could be gravitationally lensed $[4,6]$. Stimulated by these considerations we obtained medium-resolution spectroscopy of the source in order to verify the reported redshift of Q0045-3337.

\section{OBSERVATIONS AND ANALYSIS}

The object was observed at the ESO/3.6m telescope on 2007 September, 12, as a part of a spectroscopic study of high-redshift quasars (ESO proposal ID: 079.B-0304(A)). The ESO Faint Object Spectrograph and Camera [7] was mounted in long-slit spectroscopy setup with a grism yield-

*Address correspondence to this author at the Universita degli Studi dell'Insubria, via Valleggio 11 I-22100 Como, Italy; Tel: +39 (0)31 238 6311; E-mail: Roberto.Decarli@mib.infn.it ing $\lambda / \Delta \lambda \sim 400$ and a 1.2 arcsec slit. The observed wavelength range is $4100-7500 \mathrm{Ang}$. The wavelength calibration accuracy is $\sim 0.3$ Ang throughout the whole range. At $\lambda \sim 5000$ Ang, the spectral resolution is $\sim 13$ Ang. A onedimensional spectrum was integrated over a $\sim 2$ arcsec aperture. The signal-to-noise ratio at 5500 Ang is $\sim 60$.

We also obtained Johnson's V- and R-band images of the source. Fig. (1) shows the R-band field of the target. The astrometric solution was computed through the positions of GSC2 catalog sources. The target coordinates in the NASA/IPAC Extragalactic Database are marked, leaving no doubt concerning the identification of the source.

Three $800 \mathrm{~s}$ exposures were taken. The standard IRAF procedure was adopted in the data reduction. The ccdred package was employed to perform bias subtraction, flat field correction, image alignment and combination. The spectrum extraction, background subtraction and the calibrations both in wavelength and flux were performed with the doslit task in the kpnoslit package, using a He-Ar lamp and a standard spectrophotometric star as a reference. Absolute flux calibration of spectra was corrected through the photometry of field stars, by comparing corollary imaging with $\mathrm{R}$ filter to the magnitudes published in the GSC2 catalog. The measured Rband magnitude of the target (blended to the nearby galaxy) is $\sim 18.5 \pm 0.1$. Galactic extinction was accounted for [8] $(\mathrm{E}(\mathrm{B}-\mathrm{V})=0.017 \mathrm{mag})$, assuming $\mathrm{R}_{\mathrm{V}}=3.1$.

The slit was oriented along the direction between the target and the companion galaxy. The seeing was 1.4 arcsec and therefore the spectrum is the blend of these sources. On the other hand, the R-band magnitude expected for the closeby galaxy is 20.1 (assuming $\mathrm{R}-\mathrm{K}=2.6 \mathrm{mag}$ [9]), that is its contribution to the observed flux is negligible. Neither the Vand R-band images nor the spatial profile of the spectrum show any signature of the galaxy, supporting the idea that the blended spectrum is dominated by Q0045-3337. 


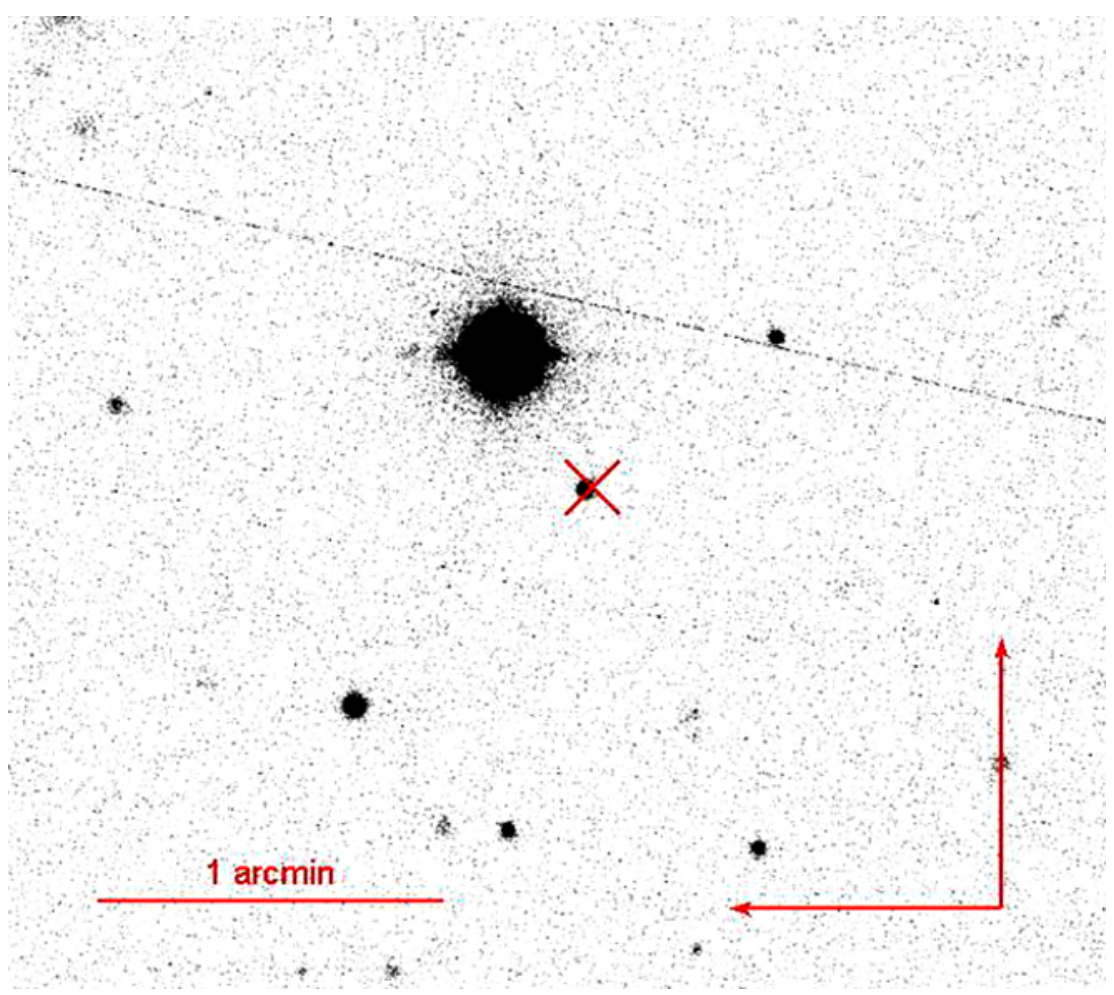

Fig. (1). The field of Q0045-3337, as imaged with the R-band filter. North is up, East is to the left-hand side of the frame. The 'x' symbol marks the corresponding coordinates in the NASA/IPAC Extragalactic Database.

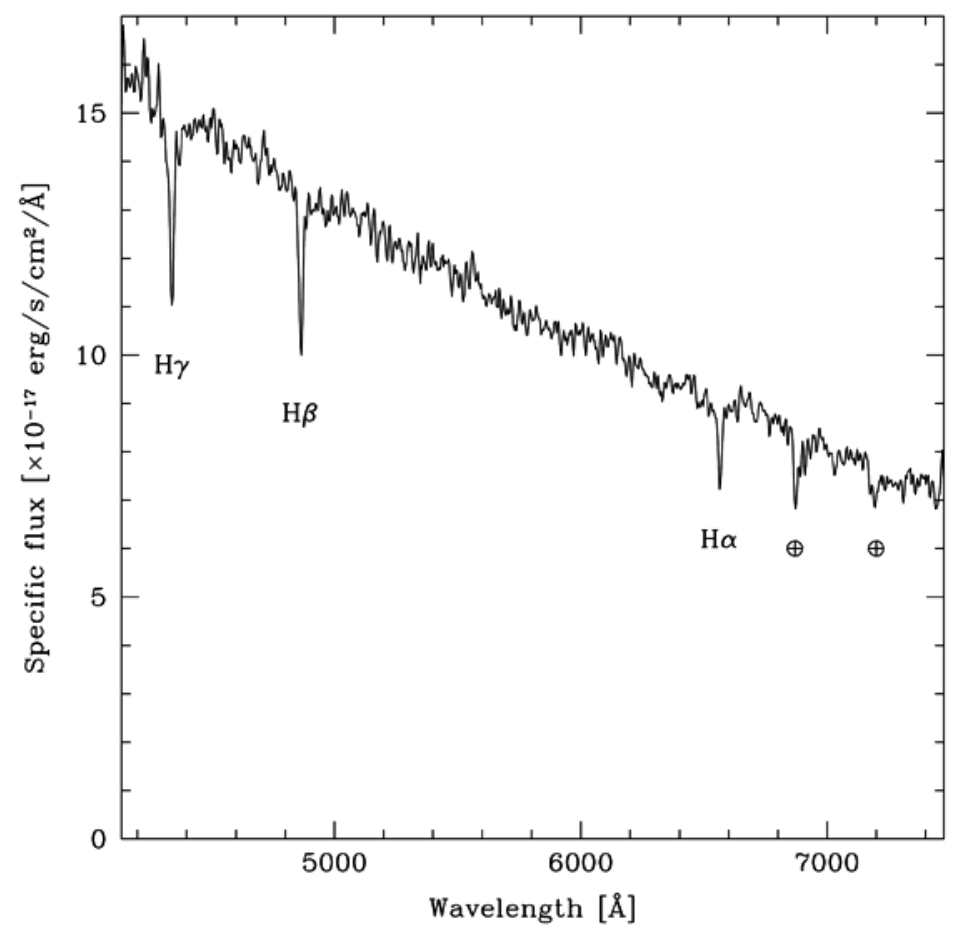

Fig. (2). The optical spectrum of the alleged quasar Q0045-3337. The rest-frame Balmer absorption lines indicate that the object is a B-type star. The Earth symbol marks the main atmospheric absorption lines.

\section{RESULTS AND CONCLUSIONS}

The optical spectrum, presented in Fig. (2), clearly shows the presence of rest-frame hydrogen absorption features over a blue continuum, indicating that it is a star of spectral type B [10]. We estimate a radial velocity of $74 \pm 16 \mathrm{~km} / \mathrm{s}$, corrected to the Galactic Center ( $b=-83.7$ degrees). Given the observed magnitude $m_{v}=18.7$, if we assume $M_{V}=-3$, typical 
of a main sequence B star, the distance of the Q0045-3337 would be $220 \mathrm{kpc}$, excluding that it is a main sequence star of the Galaxy. We consider two other possibilities: 1) that the source is a white dwarf with $8000 \mathrm{~K}$ and $\log g=7.5-8.0$; 2) a Blue Horizontal Branch (BHB) star of the halo. In the former case, assuming an absolute magnitude of $\mathrm{M}_{\mathrm{V}}=12-13$, the inferred distance would be about $150 \mathrm{pc}$. In the latter case, assuming $\mathrm{M}_{\mathrm{V}}=+0.5$, the distance would be $\sim 45 \mathrm{kpc}$. It is noticeable that, according to the Sloan Digital Sky Survey [11], the surface density of quasars candidates at g 18.7 ( 4 per magnitude in a square degree [12]) is $\sim 4$ times that of white dwarfs [13], and 40 times the surface density of BHB candidates [14].

In conclusion, we have shown that Q0045-3337 is not a quasar, but likely a white dwarf. Other similar cases were discussed in $[15,16]$.

\section{ACKNOWLEDGMENTS}

We thank Angela Iovino and Massimo Dotti for useful discussions.

\section{REFERENCES}

[1] Iovino A, Clowes RG, Shaver P. A large sample of objective prism quasar candidates. J Astrophysc suppl series 1996; 119: 265.

[2] Clowes RG. Automated quasar detection in the SGP field - A clustering study. MNRAS 1986; 218: 139.

[3] Veron-Cetty MP, Veron P. A catalogue of quasars and active nuclei: $10^{\text {th }}$ ed. Astron \& astrophyc 2001; 374: 92.

[4] Falomo R, Kotilainen JK, Scarpa R, Treves A. VLT adaptive optics imaging of QSO host galaxies and their close environment at $\mathrm{z} 2.5$ : Results from a pilot program. Astron \& astrophyc 2005; 434: 69.
[5] Falomo R, Treves A, Kotilainen JK, Scarpa R, Uslenghi M. Nearinfrared adaptive optics imaging of high-redshift quasars. J Astrophysc 2008; 673: 694.

[6] Chieregato M, Miranda M, Jetzer P. Q0045-3337: models including strong lensing by a spiral galaxy. Astron \& astrophysc 2007 ; 474: 777 .

[7] Buzzoni B, Delabre B, Dekker H, et al. The ESO Faint Object Spectrograph and Camera (EFOSC). ESO Messenger 1984; 38: 9.

[8] Schlegel, David J, Douglas P, et al. Maps of dust infrared emission for use in estimation of reddening and Cosmic Microwave Background radiation foregrounds. J Astrophysc 1998; 500: 525.

[9] Buzzoni A. Broad-band colours and overall photometric properties of template galaxy models from stellar population synthesis. Mon Not Roy Astron Soc 2005; 361: 725.

[10] Allen C.W. 1973. Astrophysical Quantities, Athlone $3^{\text {rd }}$ ed. London 1976.

[11] Adelman-McCarthy JK, Agüeros MA, Allam SS, et al. The sixth Data Release of the Sloan Digital Sky Survey. J Astrophysc Suppl Series 2008; 175: 297.

[12] Yanny B, Newberg HJ, Kent S, et al. Identification of A-colored stars and structure in the halo of the Milky Way from Sloan Digital Sky Survey Commissioning Data. J Astrophysc 2000; 540: 825.

[13] Bianchi L, Rodriguez-Merino L, Viton M, et al. Statistical properties of the GALEX-SDSS matched source catalogs, and classification of the UV sources. J Astrophysc Suppl Ser 2007; 173: 659.

[14] Pier JR. Finding BHB stars with SDSS to contrain HVC distances. J Astrophysc Suppl Ser 1999; 166: 73.

[15] Sbarufatti B, Treves A, Falomo R, Heidt J, Kotilainen J, Scarpa R. ESO Very Large Telescope Optical Spectroscopy of BL Lacertae Objects. II New redshifts, featureless objects and classification assessments. J Astron 2006; 132: 1.

[16] De Martino D, Koester D, Treves A, Sbarufatti B, Falomo R. An extremely Carbon-rich White Dwarf in the direction of the VirgoComa cluster. J Astrophysc Suppl Ser 2007; 372: 273

Received: August 29, 2008

(C) Decarli et al.; Licensee Bentham Open.

This is an open access article licensed under the terms of the Creative Commons Attribution Non-Commercial License (http://creativecommons.org/licenses/by-nc/3.0/) which permits unrestricted, non-commercial use, distribution and reproduction in any medium, provided the work is properly cited. 